\title{
Shigellosis in Nepal: 13 years review of nationwide surveillance
}

\author{
Geeta Shakya, Jyoti Acharya, Shailaja Adhikari and Nisha Rijal* (D)
}

\begin{abstract}
Background: Shigella is a major cause of gastroenteritis especially in children. In developing countries, the incidence is frequent and results are often life threatening. Changing epidemiology and emerging antibiotic resistance warrants continuous monitoring of susceptibility. The present study highlights the changing epidemiology and drug resistance patterns of Shigella isolated at different hospitals of Nepal over a period of 13 years (Jan. 2003-Dec. 2015).

Methods: This study was carried out in 12 participating laboratories. Stool specimens received at respective laboratories were processed for isolation and identification of Shigella species and confirmed by serotyping at National Public Health Laboratory. Antimicrobial resistance patterns were determined by Kirby Baeur disc diffusion test.

Results: A total of 332 isolates were identified as Shigella species of which Shigella flexneri (50\%) was the predominant serotype. Shigella dysenteriae, Shigella sonnei, Shigella boydii, and untypable Shigella spp. respectively, accounted for 28.6, $27.54,10.2,4.5$, and $6.6 \%$ of the total number. Change in prevalent serotype is noted over the years. S. dysenteriae was the prevalent species in Nepal in 2003 and 2004, but since 2005, S. flexneri remained prevalent. Majority of the isolates were recovered from children aged $1-10$ years and was statistically significant $(p=0.023)$ compared to the other age groups. High resistance among all Shigella species to the first-line drugs like ampicillin (88 \%), cotrimoxazole (76 \%), ciprofloxacin (39\%) and nalidixic acid ( $80 \%$ ) was observed; $46.1 \%$ of total isolates were multidrug resistant (MDR), and the most common MDR profile was ampicillin, nalidixic acid, and co-trimoxazole. Prevalence of MDR increased significantly in 2010 as compared to 2003. Only few Shigella isolates were resistant to ceftriaxone.
\end{abstract}

Conclusions: The study revealed S. flexneri as the predominant serogroup in Nepal. Children below 10 years were more prone to the disease. Nalidixic acid, ampicillin, co-trimoxazole, and ciprofloxacin should not be used empirically as the first-line drugs in treatment of shigellosis. Since the distribution of different species of Shigella and their antibiotic susceptibility profile may vary from one geographical location to another and may also change with time, continuous local monitoring of resistance patterns is necessary for appropriate antimicrobial therapy.

Keywords: Shigella, Antimicrobial resistance, Nepal

\section{Background}

Shigella is one of the most important etiological agents of diarrhea, in particular, dysentery commonly known as bacillary dysentery. It is a more severe form than gastroenteritis and is responsible for childhood morbidity and mortality, especially in developing countries [1-4]. Ingestion of even 100 microorganisms leads after 4-7 days to an acute diarrhea. Because of delay in humoral responses, complication and mortality rate due to shigellosis in children is higher than in other age groups [5]. A literature review concluded that of the estimated 165 million

\footnotetext{
* Correspondence: nisharijal1@gmail.com

National Public Health Laboratory, Kathmandu, Nepal
}

cases of Shigella diarrhea that occur annually in the world, $99 \%$ occur in the developing world and the remaining $1 \%$ occurs in industrialized countries. In developing countries, $69 \%$ of these episodes occur in children under 5 years of age. Moreover, of the 1.1 million deaths attributed to Shigella infections in developing countries, $60 \%$ occur in the under 5 age group in children [6].

The genus Shigella comprise of four different species which are referred to by a letter designation based on their serological antigen: serotype A-Shigella dysenteriae, Serotype B-Shigella flexneri, Serotype C-Shigella boydii and Serotype D-Shigella sonnei. The first three are 
common in developing countries while S. sonnei is common in developed countries and Iran [7].

Shifts in the prevalent serogroups have been observed in many parts of the world and so is true for Nepal. Studies conducted during1999-2002 shows high incidence of S. dysentriae infection in Nepal [8]. Studies conducted after 2002 showed prevalence of S. flexneri strains among Shigella isolates from all parts of Nepal [9-12].

According to WHO report, antimicrobial resistance pattern for Shigella varies in different parts of the world and with the time. Over the past decades, Shigella species have become progressively resistant to most widely used antimicrobials [13, 14]. Since the late 1990s, the fluoroquinolones, including ciprofloxacin, norfloxacin, and ofloxacin, have been the drug of choice for multidrugresistant Shigella infections. However, strains resistant to the first-line drugs commonly used in the treatment of shigellosis like ampicillin, cotrimoxazole, and nalidixic and even ciprofloxacin and norfloxacin has also been reported from various studies in Nepal and worldwide [9-12, 15]. Ceftriaxone has been used as a reserved antibiotic for treatment of multidrug-resistant (MDR) Shigella infection [16]. However, some studies have also reported emerging ceftriaxone resistance in Shigella. This increasing level of antimicrobial resistance in Shigella have limited the therapeutic options and complicated the treatment of shigellosis.

The purpose of the present study is to determine the trend of change in Shigella species and their antimicrobial resistance patterns over a decade for the better management of shigellosis.

\section{Methods}

\section{Surveillance sites}

Nepal is divided into five development regions. The study was carried out in 12 participating laboratories that are under Antimicrobial Resistance Surveillance network. The study was conducted in three development regions: central, eastern, and western development regions and covered six districts. In the central development region, there were five surveillance sites in Kathmandu district (National Public Health Laboratory (NPHL), Bir Hospital, Sukraraj Tropical Hospital, Kanti Children Hospital, Teaching Hospital) and two in Lalitpur district (Patan Hospital and KIST hospital). In the western development region, Western Regional Hospital and Manipal Teaching Hospital fall in Kaski district whereas Lumbini Zonal Hospital falls in Rupandehi district and United Mission Hospital falls in Palpa district.

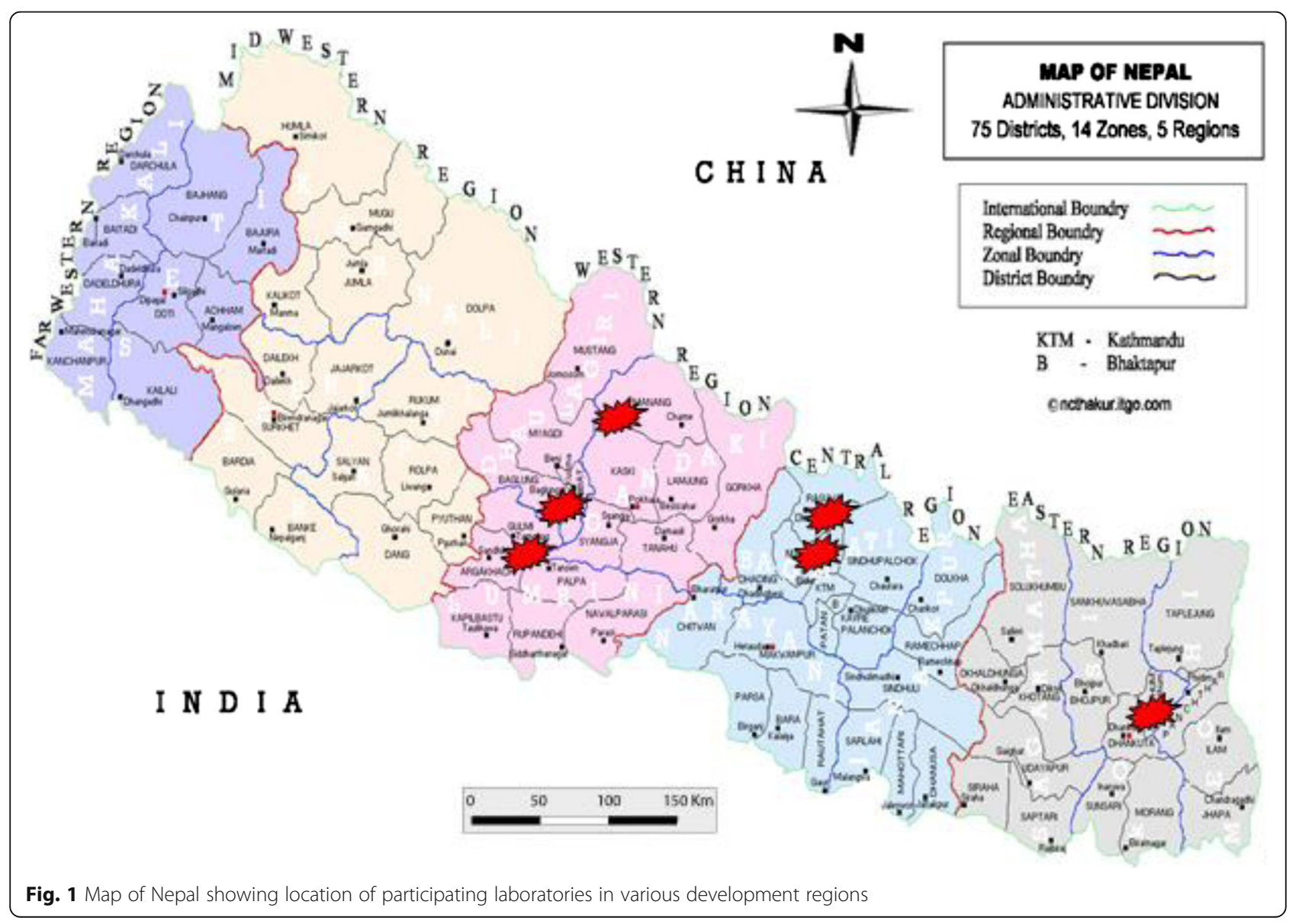




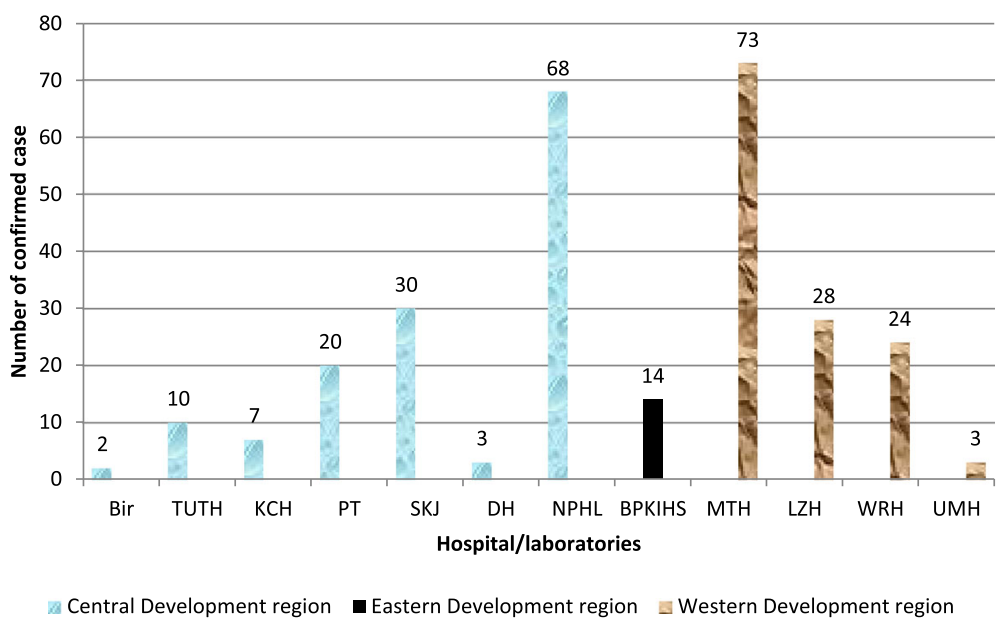

Fig. 2 Region wise reported cases of total Shigella isolates $(n=232)$

Only one site BPKIHS falls in Morang district of the eastern development region (Fig. 1).

\section{Selection of surveillance sites}

Surveillance sites were selected preferring large private/ government hospital laboratories from various development regions where the diagnostic facilities were adequate and the probability of patient inflow during outbreaks was higher.

\section{Sample population and data collection}

Diarrheal samples were collected from individual patients referring to the private or governmental laboratories. A questionnaire for each subject was filled at respective laboratories mentioning basic details of the patient, age (months), sex, location of residence, and travel. A history from all patients was also recorded, including duration of illness prior to admission to hospital (days), fever (defined as a prolonged temperature $>37.5^{\circ} \mathrm{C}$ ), abdominal discomfort, vomiting, watery diarrhea (defined as three or more loose bowel movements during a 24-h period), bloody or mucoid diarrhea (defined as $>3$ loose stools with obvious blood or mucus), estimated number of episodes of diarrhea before attending hospital, and if there was any known pretreatment with antimicrobials.

\section{Sources of Shigellae}

Isolates recovered from all 12 sites of Antimicrobial Resistance surveillance network in Nepal during 20032015 were reported to NPHL and were included in the study.

Table 1 Annual distribution of different serotypes of Shigella (2003-2015)

\begin{tabular}{|c|c|c|c|c|c|c|}
\hline Year & S. dysentriae N (\%) & S. flexneri N (\%) & S. sonnei N (\%) & S. boydii N (\%) & Unidentified Shigella spp. & Total \\
\hline 2003 & $13(52 \%)$ & $7(28 \%)$ & $2(8 \%)$ & $3(12 \%)$ & - & 25 \\
\hline 2004 & $28(63.7 \%)$ & $12(27.3 \%)$ & $2(4.5 \%)$ & $2(4.5 \%)$ & - & 44 \\
\hline 2005 & $10(19.6 \%)$ & $31(60.8 \%)$ & $6(11.8 \%)$ & $4(7.8 \%)$ & - & 51 \\
\hline 2006 & $6(26.1 \%)$ & $14(60.9 \%)$ & $2(8.7 \%)$ & $1(4.3 \%)$ & - & 23 \\
\hline 2007 & $10(27 \%)$ & $22(59.5 \%)$ & $3(8.1 \%)$ & $2(5.4 \%)$ & - & 37 \\
\hline 2008 & $2(11.8 \%)$ & $8(47.2 \%)$ & $1(5.8 \%)$ & $1(5.8 \%)$ & $5(29.4 \%)$ & 17 \\
\hline 2009 & $8(40 \%)$ & $5(25 \%)$ & $1(5 \%)$ & $1(5 \%)$ & $5(25 \%)$ & 20 \\
\hline 2010 & $5(50 \%)$ & $4(40 \%)$ & - & - & $1(10 \%)$ & 10 \\
\hline 2011 & $2(18.2 \%)$ & $5(45.4 \%)$ & $2(18.2 \%)$ & - & $2(18.2 \%)$ & 11 \\
\hline 2012 & $2(18.2 \%)$ & $6(54.5 \%)$ & $3(27.3 \%)$ & - & - & 11 \\
\hline 2013 & $5(16.1 \%)$ & $21(67.7 \%)$ & $3(9.7 \%)$ & - & $2(6.5 \%)$ & 31 \\
\hline 2014 & $1(4 \%)$ & $12(48 \%)$ & 5 (20\%) & $1(4 \%)$ & $6(24 \%)$ & 25 \\
\hline 2015 & $3(11.1 \%)$ & 19 (70.4 \%) & $4(14.8 \%)$ & - & 1 (3.7\%) & 27 \\
\hline Total & 95 (28.6 \%) & 166 (50\%) & 34 (10.2 \%) & $15(4.5 \%)$ & 22 (6.6 \%) & 332 \\
\hline
\end{tabular}




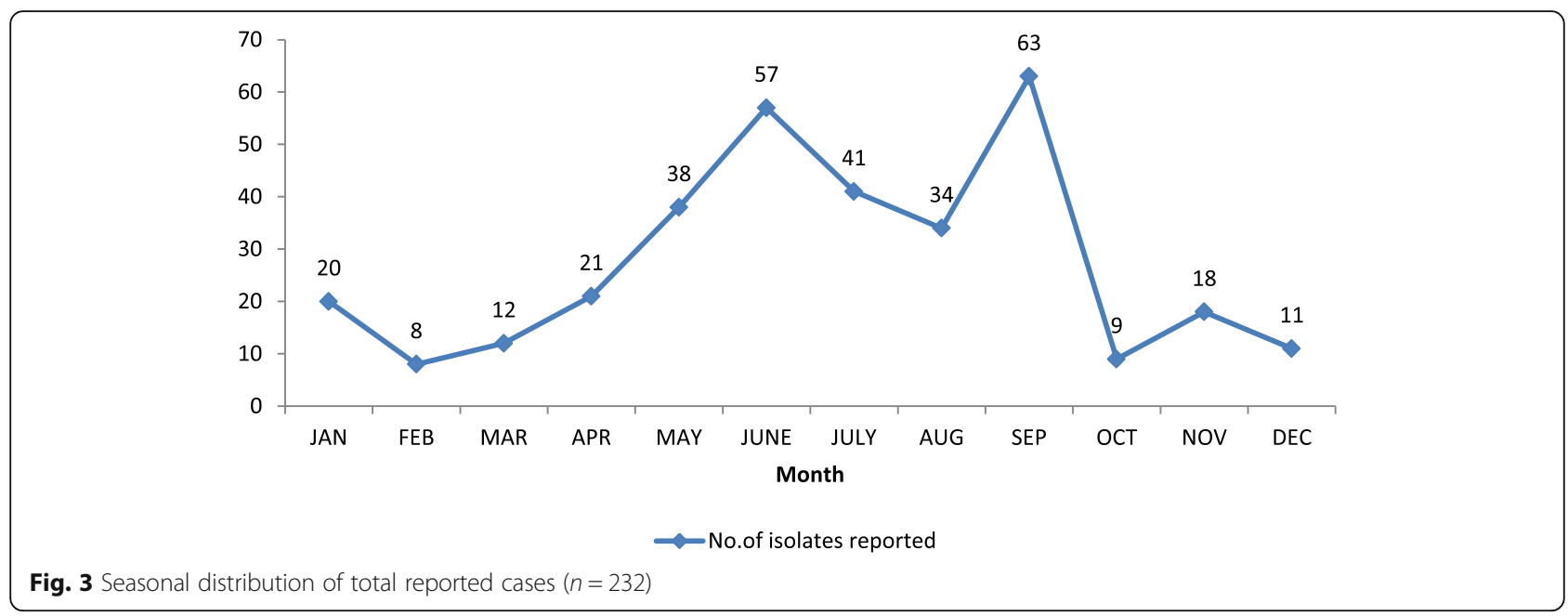

\section{Laboratory methods}

Stool specimens received at respective laboratories were cultured for enteric pathogens, including Shigella spp. by standard methods [17]. Sample was streaked onto MacConkey agar and a selective medium (either deoxycholate citrate agar or xylose lysine deoxycholate agar or Salmonella-Shigella agar) (all media by HiMedia, India) by the quadrant isolation technique [18]. The plates were incubated at $37{ }^{\circ} \mathrm{C}$ for $24 \mathrm{~h}$. Isolates were identified by Gram's staining, colony characteristics, and biochemical tests.

After identification of isolates, they were subcultured onto nutrient agar slants in screw capped tube, incubated overnight and then transported to NPHL in a cold box. All reported isolates of Shigella were re-confirmed by serotyping using commercially available antisera from Denka Seiken, Japan, and were preserved in tryptic soy broth with $20 \%$ glycerol at $-75{ }^{\circ} \mathrm{C}$ for further use. Antibiotic susceptibility test was performed for all the identified
Shigella isolates by Standard Kirby Bauer's disc diffusion technique. The antibiotics used for analysis were ampicillin (Amp-10 mcg), cotrimoxazole (Sxt-25 mcg), nalidixic acid (NA-30 mcg), ciprofloxacin (Cip-5 mcg), mecillinam $(\mathrm{Mec})$, and ceftriaxone (CRO-5 mcg).

\section{Statistical methods}

Data obtained were analyzed using SPSS software for windows version 18. Data on variables like shigella serotypes, age group, and sex were calculated as percentages and compared using chi-square test. $P<0.05$ was considered to be statistically significant. Duplicate samples were counted as one during data entry.

\section{Results}

A total of 332 Shigella isolates were reported in a period of 2003-2015 from 12 participating laboratories of Antimicrobial Resistance surveillance network in Nepal. The

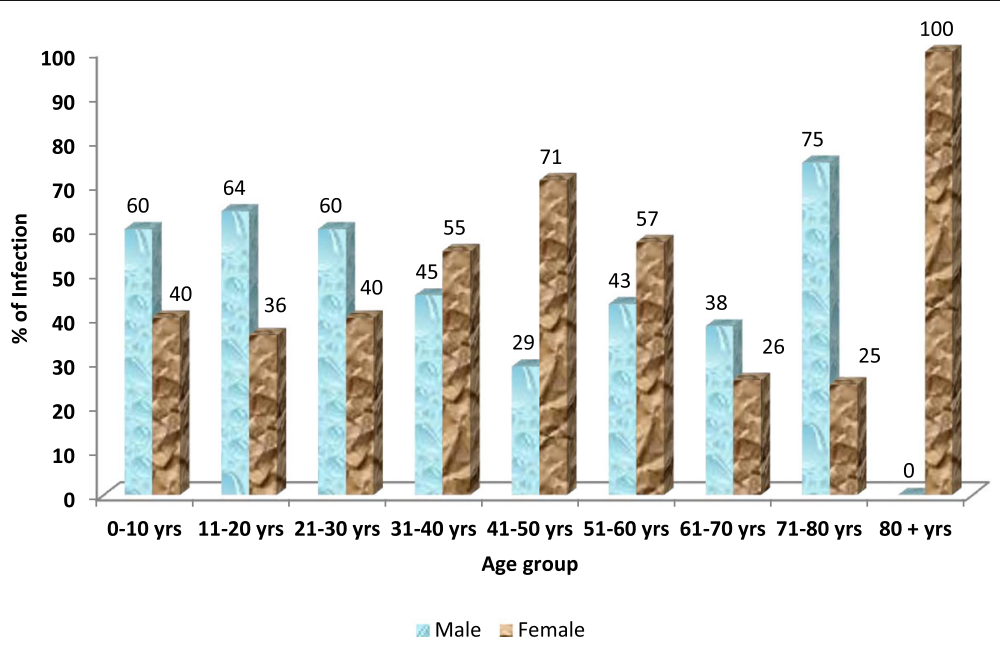

Fig. 4 Percentage of laboratory-confirmed Shigella infection reported to NPHL, by age group and sex ( $n=196$ with age and sex information reported) 
Table 2 Antimicrobial susceptibility profile of various species of Shigella (2003-2015)

\begin{tabular}{|c|c|c|c|c|c|c|c|c|}
\hline \multirow[b]{2}{*}{ Year } & \multicolumn{8}{|c|}{$N(\%)$ resistance to } \\
\hline & Species & Number tested & Ampicillin & Ciprofloxacin & Nalidixic acid & Cotrimoxazole & Mecillinam & Ceftriaxone \\
\hline \multirow[t]{4}{*}{2003} & S. dysentriae & 13 & $11(85)$ & $8(62)$ & $10(77)$ & $9(69)$ & $5(38)$ & 0 \\
\hline & S. flexneri & 7 & $7(100)$ & 0 & 0 & $6(86)$ & $2 / 6(33)^{a}$ & 0 \\
\hline & S. boydii & 3 & $2(66)$ & 0 & $1(33)$ & $2(66)$ & $2(66)$ & 0 \\
\hline & S. sonnei & 2 & $1(50)$ & 0 & $2(100)$ & $2(100)$ & $1(50)$ & 0 \\
\hline \multirow[t]{4}{*}{2004} & S. dysentriae & 28 & $25(89)$ & $13(46)$ & $14(50)$ & $18(64)$ & $20 / 27(74)^{a}$ & 0 \\
\hline & S. flexneri & 12 & $9(75)$ & $6(50)$ & $6(50)$ & $8(67)$ & $4(33)$ & 0 \\
\hline & S. boydii & 2 & $1(50)$ & 0 & 0 & $1(50)$ & 0 & 0 \\
\hline & S. sonnei & 2 & 0 & 0 & $2(100)$ & $2(100)$ & 0 & 0 \\
\hline \multirow[t]{4}{*}{2005} & S.dysentriae & 10 & $8(80)$ & $6 / 9(66)^{a}$ & $7(70)$ & $8(80)$ & $2 / 8(25)^{a}$ & 0 \\
\hline & S. flexneri & 31 & $18 / 30(60)^{a}$ & 0 & $17 / 28(59)^{a}$ & $21(68)$ & $13(42)$ & 0 \\
\hline & S. boydii & 6 & $4(66)$ & 0 & $3(50)$ & 0 & $4(66)$ & 0 \\
\hline & S. sonnei & 4 & $1(25)$ & 0 & $2(50)$ & $2(50)$ & 0 & 0 \\
\hline \multirow[t]{4}{*}{2006} & S. dysentriae & 6 & $4 / 5(80)^{a}$ & 0 & $4 / 5(80)^{a}$ & $2 / 5(40)^{a}$ & $4(66)$ & 0 \\
\hline & S. flexneri & 14 & $8 / 13(62)^{a}$ & $3 / 12(25)^{a}$ & $4 / 10(40)^{a}$ & $7 / 12(58)^{a}$ & $14(100)$ & 0 \\
\hline & S. boydii & 2 & 0 & 0 & 0 & 0 & 0 & 0 \\
\hline & S. sonnei & 1 & S & $\mathrm{R}$ & $\mathrm{R}$ & $S$ & $S$ & S \\
\hline \multirow[t]{4}{*}{2007} & S. dysentriae & 10 & $5 / 6(83)^{a}$ & $4(40)$ & $2(20)$ & $2(20)$ & $10(100)$ & 0 \\
\hline & S. flexneri & 22 & $17 / 21(81)^{a}$ & $12 / 21(57)^{a}$ & $21(95)$ & $19 / 21(90)^{a}$ & $22(100)$ & $2 / 20(10)^{a}$ \\
\hline & S. boydii & 3 & $3(100)$ & 0 & 0 & 0 & $3(100)$ & 0 \\
\hline & S. sonnei & 2 & 0 & 0 & 0 & 0 & $2(100)$ & 0 \\
\hline \multirow[t]{4}{*}{2008} & S. dysentriae & 2 & 0 & $2(100)$ & $2(100)$ & 0 & $2(100)$ & 0 \\
\hline & S. flexneri & 8 & $8(100)$ & $3 / 5(60)^{a}$ & $8(100)$ & $4 / 5(80)^{a}$ & $8(100)$ & 0 \\
\hline & S. sonnei & 2 & $2(100)$ & 0 & $2(100)$ & 0 & $2(100)$ & 0 \\
\hline & Shigella spp. & 5 & $3(60)$ & $1 / 4(25)^{a}$ & $2 / 4(50)^{a}$ & $3 / 4(75)^{\mathrm{a}}$ & $5(100)$ & $1 / 3(33)^{a}$ \\
\hline \multirow[t]{4}{*}{2009} & S. dysentriae & 8 & $8(100)$ & $2(25)$ & $7(88)$ & $7(88)$ & $8(100)$ & 0 \\
\hline & S. flexneri & 6 & $4 / 5(80)^{a}$ & $6(100)$ & $4 / 5(80)^{a}$ & $6(100)$ & $6(100)$ & $2(33)$ \\
\hline & S. sonnei & 1 & $\mathrm{R}$ & $S$ & $\mathrm{R}$ & $\mathrm{S}$ & $\mathrm{R}$ & $S$ \\
\hline & Shigella spp. & 5 & $3(60)$ & $3(60)$ & $4(80)$ & $4(80)$ & $5(100)$ & $2(40)$ \\
\hline \multirow[t]{3}{*}{2010} & S. dysentriae & 5 & $5(100)$ & $1(20)$ & $5(100)$ & $5(100)$ & - & 0 \\
\hline & S. flexneri & 4 & $4(100)$ & $1(25)$ & $4(100)$ & $4(100)$ & - & 0 \\
\hline & Shigella spp. & 1 & $\mathrm{R}$ & S & $\mathrm{R}$ & $\mathrm{R}$ & - & $S$ \\
\hline \multirow[t]{4}{*}{2011} & S. dysentriae & 2 & $2(100)$ & $1(50)$ & $1(50)$ & $2(100)$ & - & 0 \\
\hline & S. flexneri & 5 & $3(60)$ & 0 & 0 & $3 / 4(75)^{a}$ & - & 0 \\
\hline & S. sonnei & 2 & $1(50)$ & 0 & $2(100)$ & 0 & - & 0 \\
\hline & Shigella spp. & 2 & $2(100)$ & 0 & $2(100)$ & $2(100)$ & - & 0 \\
\hline \multirow[t]{3}{*}{2012} & S. dysentriae & 4 & $4(100)$ & 0 & $3(75)$ & $3(75)$ & - & 0 \\
\hline & S. flexneri & 7 & $7(100)$ & $4 / 6(66)^{a}$ & $3 / 4(75)^{a}$ & $4 / 6(67)^{a}$ & - & $2 / 6(33)^{a}$ \\
\hline & S. sonnei & 3 & 0 & 0 & $1(33)$ & $3(100)$ & - & 0 \\
\hline \multirow[t]{4}{*}{2013} & S. dysentriae & 5 & $5(100)$ & 0 & $4(80)$ & $3(60)$ & - & 0 \\
\hline & S. flexneri & 19 & $15 / 16(93)^{a}$ & $12 / 17(71)^{a}$ & $16 / 18(89)^{a}$ & $14(74)$ & - & $5 / 18(27)^{a}$ \\
\hline & S. sonnei & 3 & $3(100)$ & $2(66)$ & $2(66)$ & $2(66)$ & - & 0 \\
\hline & Shigella spp. & 3 & $3(100)$ & 0 & $3(100)$ & $3(100)$ & - & 0 \\
\hline
\end{tabular}


Table 2 Antimicrobial susceptibility profile of various species of Shigella (2003-2015) (Continued)

\begin{tabular}{|c|c|c|c|c|c|c|c|c|}
\hline \multirow[t]{5}{*}{2014} & S. dysentriae & 1 & S & R & $S$ & S & - & $S$ \\
\hline & S. flexneri & 12 & $7 / 11(64)^{a}$ & $8(66)$ & $11(92)$ & $7(58)$ & - & 0 \\
\hline & S. sonnei & 5 & $3(60)$ & $5(100)$ & $5(100)$ & $5(100)$ & - & 0 \\
\hline & S. boydii & 1 & S & R & $\mathrm{R}$ & R & - & S \\
\hline & Shigella spp. & 6 & $6(100)$ & $1 / 5(20)^{\mathrm{a}}$ & $3 / 4(75)^{a}$ & $3(50)$ & - & 0 \\
\hline \multirow[t]{4}{*}{2015} & S. dysentriae & 3 & 0 & 0 & $3(100)$ & $2(75)$ & - & 0 \\
\hline & S. flexneri & 19 & $16 / 18(88)^{\mathrm{a}}$ & $9(47)$ & $15(79)$ & $17(89)$ & - & $3(16)$ \\
\hline & S. sonnei & 4 & $3(75)$ & $4(100)$ & $4(100)$ & $4(100)$ & - & 0 \\
\hline & Shigella spp. & 1 & R & S & $\mathrm{R}$ & R & - & S \\
\hline
\end{tabular}

${ }^{a}$ The number in which antibiotics were incorporated, if less than the total number of isolates

highest number was reported in 2005. Peak isolation was seen in post monsoon season, i.e., September (Fig. 2).

Shigella dysenteriae was the prevalent species in Nepal in the years 2003-2004 but since 2005, S. flexneri replaced it and became the most prevalent species. A marked increase in the number of $S$. sonnei $(N=6)$ was noticed in the year 2005 which is highest reported till date. In contrast, no isolate of $S$. boydii was reported since 2009 till 2014 where one isolate was reported. Since 2008, few cases of unidentified Shigella isolates were also reported (Table 1).

Region wise distribution of reported isolates showed that 18 isolates of Shigella were reported from one laboratory in the eastern development region, 188 isolates were reported from seven participating laboratories in the central development region, and 126 were reported from four participating laboratories in the western development region (Fig. 3).

Of the total, $24.5 \%$ of cases were demonstrated in children below 10 years followed by $21-30$ years age group $(22.9 \%)$ and $11-20$ years age group (18.5\%). Significant difference was not noted $(p=0.059)$ in age wise distribution of cases over the years. Shigella infection was reported higher among male in age groups below 30 years and 71-80 years but higher cases were reported in females of age groups 31-70 years and $80+$ years (Fig. 4).

The antimicrobial susceptibility profile of Shigella isolates in the years 2003-2015 is shown in Table 2. Different serotypes of Shigella exhibited various resistance patterns. S. dysentriae showed varying resistance to ampicillin (80$100 \%)$, co-trimoxazole (20-100\%), and nalidixic acid (20-100 \%). Likewise, S. flexneri also exhibited varying resistance to ampicillin (80-100\%), co-trimoxazole (58-100\%), and nalidixic acid (0-100 \%). Until 2003, only $S$. dysentriae strains exhibited resistance to ciprofloxacin; however, $50 \%$ of the S. flexneri isolates were resistant to ciprofloxacin by 2004. By 2012, S. dysentriae strains were found susceptible to ciprofloxacin in contrast to $S$. flexneri, which exhibited highest resistance. Among S. sonnei and S. boydii serotypes, resistance to ampicillin, cotrimoxazole, and nalidixic acid kept varying. Only three S. sonnei isolates were found resistant to ciprofloxacin while no ciprofloxacin-resistant $S$. boydii was reported. All the serotypes of Shigella were $100 \%$ resistant to mecillinam since 2007; so by 2010, mecillinam was no longer included in antimicrobial testing of Shigella. Among the serotypes, resistance to ceftriaxone was first reported in S. flexneri (10$33 \%)$ followed by non-typable Shigella spp. with around $40 \%$ resistance; $22.2 \%$ of the total isolates tested were resistant to all three antimicrobials. Figure 5 demonstrates the cumulative antibiogram of various species of Shigella.

The prevalence of multidrug-resistant (resistance to two or more classes of antibiotics) Shigella isolates is demonstrated in Fig. 5. The prevalence of MDR increased significantly in 2010 as compared to 2003. On the basis of their pattern of antimicrobial resistance to the six antibiotics used in the study, the reported Shigellae were grouped into seven phenotypes (Table 3). Among the Shigella serotypes, high prevalence of MDR was observed in S. dysentriae isolates occupying $52 \%$ of the total isolates.

\section{Discussion}

Shigellosis still accounts for a significant proportion of mortality and morbidity, especially in developing countries [19]. The present study, covering 13 years surveillance (2003-2015), demonstrates the changing serotype and trend of antimicrobial resistance of Shigella isolates in Nepal.

An analysis of serotypes revealed that $S$. dysenteriae was the prevalent species in Nepal in the years 1999-2004 but a change in serotype in Shigella species was noticed during 2005. The changing patterns in the distribution of Shigella serogroups and serotypes have been reported from time to time [20-23]. In our study, S. flexneri remained the most prevalent serotype in Nepal since 2005 which is in accordance with many recent studies in Nepal and other developing countries [9, 15, 17, 24-27] but dissimilar to some other studies [28-30]. It has been hypothesized that these changes may be due to varying 


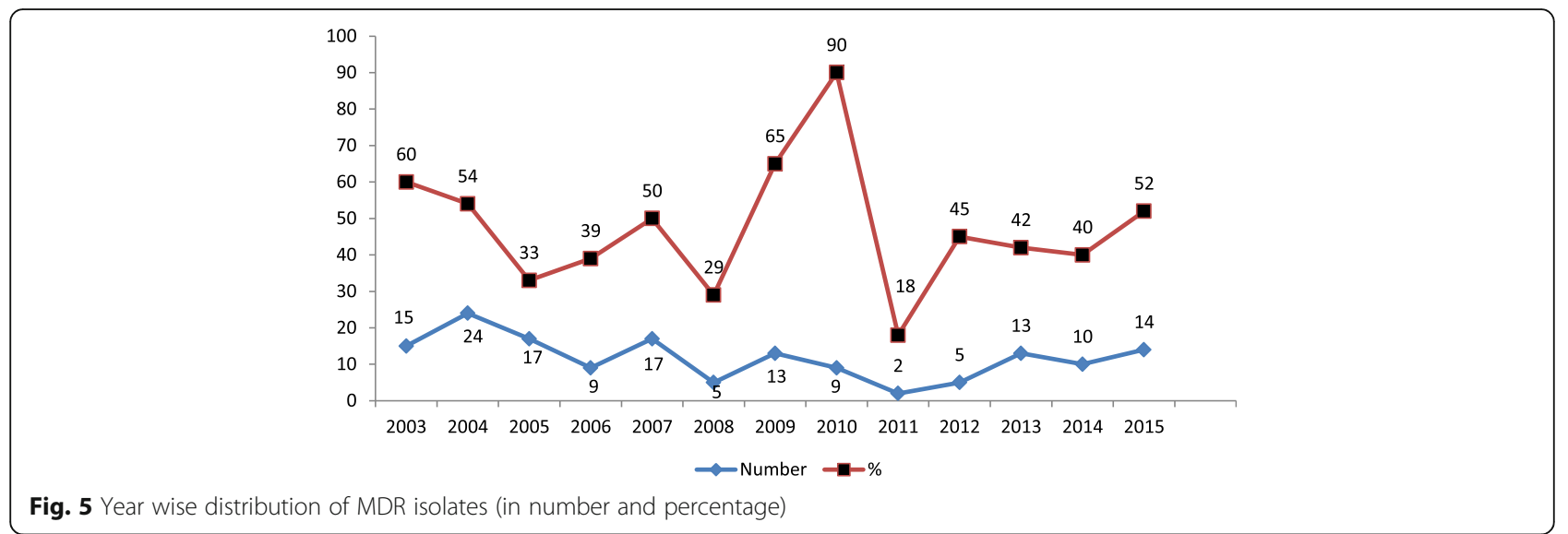

behavourial and environmental factors such as reduction of overall malnutrition, use of safe drinking water instead from tubewells or pipes, and improved sanitary facilities for defecation [31].

In our study, like in other studies, the seasonal tendency of shigellosis was summer-monsoon $[9,15]$. This may be because during this period, there is a climatic variation due to which the sources of drinking water gets rapidly contaminated. Males were more commonly infected as compared to females as in other studies made by Khan et al. and Taneja [15, 32]. This male preponderance may be due to greater chances of exposure to male population who go out of the house much frequently than females for eating food from restaurants and street vendors. The susceptible age group in our study, like in other studies, was below 10 years $[12,15,25]$. In our study, very few cases were reported from children below 5 years. This may be because the laboratories in the surveillance network are limited; many cases may be referred to private institutions which are not included in the surveillance network. Besides that, other factors like incomplete information on age (16\% missing) available from participating laboratories, inclusion of only one children's hospital in the surveillance and low Shigella isolation rate may have contributed to under reporting of true number of victims.
Treatment of shigellosis depends on appropriate antimicrobial therapy. A variety of antibiotics are effective for treatment of shigellosis, although options are becoming limited due to globally emerging drug resistance. The present study also shows high resistance among all Shigella species to the first line low cost drugs commonly used in the treatment of shigellosis like Ampicillin, Cotrimoxazole and Nalidixic acid. Considering resistance pattern in different groups, S. flexneri and S. dysentriae, the two most common serotypes revealed similar pattern in resistance with slightly higher range in S. flexneri. However, the difference was not statistically significant. Similar studies conducted by Khan et al. reveals that $S$. flexneri exhibited increased resistance to ampicillin and nalidixic acid as compared to $S$. dysentriae whereas high resistance was observed against ciprofloxacin and cotrimoxazole in S. flexneri as compared to $S$. dysentriae [15]. This finding however does not agree with some previous studies done by Wilson et al. and Kansakar et al. [9, 12] in Nepal which demonstrates high resistance in S. dysentriae. Among remaining serotypes, untypable Shigella spp. exhibited high resistance to all antimicrobials except ciprofloxacin as compared to S. sonnei and S. boydii. S. boydii exhibited least resistance to all antimicrobials tested. This finding is also in accordance to studies made by Khan

Table 3 Multidrug-resistant phenotypes of Shigella

\begin{tabular}{|c|c|c|c|c|c|}
\hline \multirow[t]{2}{*}{ MDR phenotype } & \multicolumn{5}{|c|}{ Number (\%) of isolates exhibiting resistance } \\
\hline & S. dysentriae & S. flexneri & S. boydii & S. sonnei & Shigella spp \\
\hline Amp/Cip or NA/Mec & $1(1.9 \%)$ & $3(5.2 \%)$ & $2(50 \%)$ & - & - \\
\hline $\mathrm{Amp} / \mathrm{Mec} / \mathrm{Ts}$ & $5(9.4 \%)$ & $3(5.2 \%)$ & - & - & - \\
\hline Mec/Cip or NA/Ts & $2(3.8 \%)$ & $3(5.2 \%)$ & - & $1(10 \%)$ & - \\
\hline Amp/NA or Cip/Ts & $25(47.2 \%)$ & $36(62.1 \%)$ & $1(25 \%)$ & $9(90 \%)$ & $3(50 \%)$ \\
\hline Amp/NA or Cip/Ts/Mec & $20(37.7 \%)$ & $5(8.6 \%)$ & - & - & $3(50 \%)$ \\
\hline Amp/NA or $\mathrm{Cip} / \mathrm{Ts} / \mathrm{Mec} / \mathrm{CRO}$ & - & $1(1.7 \%)$ & $1(25 \%)$ & - & - \\
\hline Amp/NA or Cip/Ts/CRO & - & $7(11.9 \%)$ & - & - & - \\
\hline Total number MDR (\%) & $53(55.8 \%)$ & $58(34.9 \%)$ & $4(26.6 \%)$ & $10(29.4 \%)$ & $6(27.3 \%)$ \\
\hline
\end{tabular}


et al. [15] but contradictory to findings by Kansakar et al. [9] which reveals $S$. sonnei as the most susceptible serotype. Emerging resistance to ceftriaxone in S. dysentriae, S. flexneri, and Shigella spp. is noted which raises concern for the use of third-generation cephalosporins as last therapy of treatment.

This difference in antimicrobial resistance among various serotypes may be attributed to change in time period of the studies. Studies conducted during 2004/2005 showed contradictory result in terms of prevalent serotype and resistance profile among serotypes as compared to that of $2013 / 2014$ which seems obvious.

MDR (resistance to three or more than three antibiotics) was found in $46.1 \%$ of total isolates, and the most common MDR profile was ampicillin, nalidixic acid, and cotrimoxazole. This finding is very low as compared to $90.2 \%$ reported by Reema et al. However, the MDR profile is similar [33].

\section{Limitations}

Data represented from our 12 surveillance sites may not be representative of the sick population at large in the community. Lack of total number of reported cases and inadequate data management systems also limited the results of our study.

\section{Conclusions}

With increasing resistance to first-line agents along with ciprofloxacin, only ceftriaxone remains the drug of choice; however, increasing MDR along with resistance to thirdgeneration cephalosporins as last resort creates an alarming situation warranting proper usage of antimicrobial agents and its continuous monitoring.

\section{Abbreviations \\ Amp: Ampicillin; BPKIHS: B. P. Koirala Institute of Health Sciences; Cip: Ciprofloxacin; CRO: Ceftriaxone; KIST: KIST Medical College and Teaching Hospital; MDR: Multidrug resistant; Mec: Mecillinam; NA: Nalidixic acid; NPHL: National Public Health Laboratory; Ts: Cotrimoxazole; WHO: World Health Organization}

\section{Acknowledgements}

We acknowledge all the laboratory staffs of the participating laboratories for their contribution in laboratory diagnosis of Shigella in their respective units. We are thankful for their invaluable support.

\section{Funding}

Not applicable.

\section{Availability of data and materials}

Please contact author for data requests.

\section{Authors' contributions}

GS critically reviewed the manuscript and approved the final version of the manuscript. JA conceived and designed the research. SA analyzed and interpreted the data. NR finalized and drafted the manuscript. All authors have read and approved the final manuscript.

\section{Competing interests}

The authors declare that they have no competing interests.

\section{Ethics approval and consent to participate}

At the time of enrollment, verbal consent was taken from the caregivers or guardians on behalf of the patients. Parents or guardians were assured about the non-disclosure of information collected from them and were also informed about the use of data for analysis and using the results for improving patient care activities as well as publication without disclosing the name or identity of their children. The consent to utilize the data was obtained from respective participating laboratories who reported the cases, and the laboratories have no objection in its publication. The ethical review board of NPHL has approved the publication of this data.

Received: 17 March 2016 Accepted: 21 October 2016

Published online: 04 November 2016

\section{References}

1. Raqib R, et al. Improved outcome in shigellosis associated with butyrate induction of an endogenous peptide antibiotic. Proc Natl Acad Sci USA. 2006;103(24):9178-83.

2. Sur D, et al. Shigellosis: challenges and management issues. Indian J Med Res. 2004;120(5):454-62.

3. Ashkenazi S. Shigella infections in children: new insights. Semin Pediatr Infect Dis. 2004;15(4):246-52.

4. Ghaemi EO, Aslani MM, Moradi AV, Dadgar T, Livani S, Mansourian AR, et al. Epidemiology of Shigella-associated diarrhea in Gorgan, North of Iran. Saudi J Gastroenterol. 2007;13(3):129-32.

5. Agence Francaise de Sacurite Sanitaire des Produits de Sante. Antibiotic treatment of Shigella sonnei gastroenteritis. Presse Med. 2004;33(21):1538-45.

6. Kotloff KL, Winickoff JP, Ivanoff B, Clemens JD, Swerdlow DL, Sansonetti PJ, et al. Global burden of Shigella infections: implications for vaccine development and implementation of control strategies. Bull World Health Organ. 1999;77:651-66.

7. Farshad, S, R. Sheikhi, A. Japoni, E. Basiri and A. Alborzi. Characterization of Shigella strains in Iran by plasmid profile analysis and PCR amplification of ipa genes. J. Clin. Microbiol. 2006; 44:2879-2883.

8. Shrestha CD, Malla S and Maharjan L. Multi drug resistant Shigella species in Nepal, a retrospective study conducted at National Public Health Laboratory (NPHL), 1999 to 2002. Journal of Nepal Health Research Council Vol.4 No.1 April, Pg 51-55

9. Kansakar P, Malla S, Ghimire GR. Shigella isolates of Nepal: changes in the incidence of Shigella subgroups and trends of antimicrobial susceptibility pattern. Kathmandu University Medical Journal 2007, Vol. 5, No. 1, Issue 17, 32-37

10. Sur D, Niyogi SK, Sur S, Datta KK, Takeda Y, Nair GB, et al. Multi drug resistant Shigella dysenteriae type 1: forerunners of a new epidemic strains in eastern India? Emerg Infect Dis. 2003;9:404-5.

11. ICDDR,B. Antimicrobial resistance surveillance for selected infectious disease pathogens in Nepal, 1999-2003. Final report. 2003, 32-7 p.

12. Wilson G, Easow JM, Mukhopadhyay C, Shivananda PG. Isolation and antimicrobial susceptibility of Shigella from patients with acute gastroenteritis in western Nepal. India J Med Res. 2006:123:145-50.

13. Sack BR, Rahman M, Yunus M, Khan HE. Antimicrobial resistance in organisms causing diarrheal disease. Clin Infect Dis. 1997;24 Suppl 1:S102-5.

14. Bennish ML, Salam MA, Hossain MA, Myaux J, Khan EH, Chakraborty J, et al. Antimicrobial resistance of Shigella isolates in Bangladesh, 1983-1990: increasing frequency of strains multiply resistant to ampicillin, trimethoprimsulfamethoxazole, and nalidixic acid. Clin Infect Dis. 1992;14:1055-60.

15. Khan $S$, Singh $P$, Ansari M, Asthana A. Isolation of Shigella species and their resistance patterns to a panel of fifteen antibiotics in mid and far western region of Nepal. Asian Pac J Trop Dis. 2014;4(1):30-4.

16. Goel N, Wattal C, Kaul D, Khanna VK. Emergence of ceftriaxone resistant Shigella. Indian j paediatr. 2013;80(1):70-1.

17. Collee J.G, Fraser AG, Marmion BP and Simmons A. Mackie and Mc Cartney practical microbiology, 14th edition, 1996 Churchill Livingstone, Longman Group, UK.

18. Hoque L, Kamal SMM, Ahmed Z. Isolation, identification and antimicrobial sensitivity pattern of bacterial isolates from tracheal aspirate of ICU patients of central Dhaka, Bandladesh. Int j res appl nat soc sci. 2013;1(7):11-6.

19. Ranjbar R, Dallal MM, Pourshafie MR. Epidemiology of shigellosis with special reference to hospital distribution of Shigella strains in Tehran. Iran I Clin Infect Dis. 2008:3(1):35-8.

20. Niyogi SK, Mitra U, Dutta P. Changing patterns of serotypes and antimicrobial susceptibilities of Shigella species isolated from children in Calcutta, India. Jpn J Infect Dis. 2001;54:121-2. 
21. Sonawala M, Saraswathi K, Deodhar LP. Serogroup prevalence of Shigellae in Bombay. J Postgrad Med. 1995;41:104-6.

22. Jesudason MV. Shigella isolation in Vellore, South India (1997-2001). Indian J Med Res. 2002:115:11-3.

23. Zaman K, Yunus M, Baqui AH, Hossain KMB. Surveillance of shigellosis in rural Bangladesh: a 10 years review. J Pak Med Assoc. 1991;41:75-8.

24. Bhattacharya S, Khanal B, Bhattarai NR, Das ML. Prevalence of Shigella species and their antimicrobial resistance patterns in Eastern Nepal. J Health Popul Nutr. 2005;23(4):339-42.

25. Savadkoohi R.B, Ahmadpour-Kacho. M, Prevalence of Shigella species and their antimicrobial resistance patterns at Amirkola Children's Hospital, North of Iran. Iran J Ped June 2007, Vol 17: 2,118-122

26. Srinivasa H, Baijayanti M, Raksha Y. Magnitude of drug resistant shigellosis: a report from Bangalore. Indian J Med Microbiol. 2009;27(4):358-60.

27. Bhattacharya D, Sugunan AP, Bhattacharjee H, Thamizhmani R, Sayi DS, Thanasekaran K, et al. Antimicrobial resistance in Shigella-rapid increase \& widening of spectrum in Andaman Islands, India. Indian J Med Res. 2012;135:365-70

28. Urvashi, Saxena S, Dutta R. Antimicrobial resistance pattern of Shigella species over 5 years at a tertiary-care teaching hospital in north India. J Health Popul Nutr 2011; 29 (3): 292-295.

29. Dutta S, Dutta D, Dutta P, Matsushita S, Bhattacharya SK, Yoshida SI. Shigella dysenteriae serotype 1, Kolkata, India. Emerg Infect Dis. 2003;9(11):1471-4.

30. Thapa BR, Ventkateswarlu K, Malik AK, Panigrahi D. Shigellosis in children from north India: a clinicopathological study. J Trop Pediatr. 1995;41(5):303-7.

31. Faruque AS, Teka T, Fuchs GJ. Shigellosis in children: a clinico-epidemiological comparison between Shigella dysenteriae type I and Shigella flexneri. Ann Trop Paediatr. 1998;18(3):197-201.

32. Taneja N. Changing epidemiology of Shigella and emergence of ciprofloxacin resistant strains Shigellae in India. J Clin Microbiol. 2007;45(2):678-9.

33. Nath R, Saikia L, Choudhury G, Sharma D. Drug resistant Shigella flexneri in \& around Dibrugarh, north-east India, Indian J Med Res 137, January 2013: 183-18

\section{Submit your next manuscript to BioMed Central and we will help you at every step:}

- We accept pre-submission inquiries

- Our selector tool helps you to find the most relevant journal

- We provide round the clock customer support

- Convenient online submission

- Thorough peer review

- Inclusion in PubMed and all major indexing services

- Maximum visibility for your research

Submit your manuscript at www.biomedcentral.com/submit

) Biomed Central 